\title{
Early Detection of Fatigue Damage Using Escort Distributions of Ultrasonic Data Sequences *
}

\author{
Dheeraj Sharan Singh \\ dss240@psu.edu
}

\author{
Shalabh Gupta \\ szg107@psu.edu
}

Subhadeep Chakraborthy
szc138@psu. edu

The Pennsylvania State University

University Park, PA 16802

\author{
Asok Ray \\ axr2@psu.edu
}

\begin{abstract}
This paper presents real-time detection of fatigue damage in mechanical structures using ultrasonic sensing methodology. The data-driven pattern identification method for anomaly detection is based on the tools derived from Statistical Mechanics and Symbolic Dynamics. The concept of Escort Distributions has been used to identify the behavioral patterns changes in complex systems due to gradual evolution of anomalies. The real-time information of evolving fatigue damage provides early warnings of forthcoming catastrophic failures. The anomaly detection method has been experimentally validated on poly-crystalline alloys using ultrasonic data generated from a fatigue damage testing apparatus.
\end{abstract}

\section{INTRODUCTION}

Damage due to fatigue phenomenon is one of the most commonly encountered sources of structural degradation in complex electromechanical systems. Detection of fatigue damage at an early stage is essential because the accumulated damage could potentially cause catastrophic failures, leading to loss of life and expensive equipment. In the current stateof-the-art, direct measurements of fatigue damage at an early stage (e.g., crack initiation) are not feasible due to lack of analytical models and sensing devices. Specifically, random distribution of flaws in identically manufactured structural components leads to different behavioral patterns of fatigue damage evolution. Consequently, the analysis of time series data from the available sensors is essential for monitoring the fatigue damage evolution in real time.

Several techniques based on various sensing devices (e.g., ultrasonics, acoustic emission, and eddy currents) have been proposed in recent literature for fatigue crack monitoring [1][2]. Impedance of ultrasonic signals has been shown to be sensitive to small microstructural changes during the early stages of fatigue damage evolution [3]. Technical literature abounds with diverse techniques of pattern recognition [4]. Anomaly detection using symbolic dynamic filtering $(S D F)$ is a pattern recognition method that has been recently developed [5] and a comparative evaluation of this novel analytical method shows its superior performance relative to other existing pattern recognition tools in terms of early detection of small changes in dynamical systems [6] and robustness to noisy environments [7].

This paper presents fatigue damage monitoring in polycrystalline alloys using the application of the pattern recog-

\footnotetext{
$\star$ This work has been supported in part by This work has been supported in part by the U.S. Army Research laboratory and the U.S. Army Research Office under Grant No. W911NF-07-1-0376.
}

nition tools of $S D F$ and the concept of escort distributions from Statistical Mechanics. To this end, the concepts have been experimentally validated on a computer-controlled fatigue damage testing apparatus that is equipped with ultrasonic transducers and an optical microscope. The main contributions of this paper are listed below.

1) Ultrasonic data analysis using symbolic dynamic filtering $(S D F)$ for real-time information extraction

2) Identification of behavioral patterns for detection of gradually evolving anomalies based on the statistical mechanical concept of escort distributions [8]

3) Experimental validation of the techniques on a fatigue damage testing apparatus using polycrystalline alloys.

\section{Behavioral PATtern Identification}

The study of dynamical systems using the techniques of Statistical Mechanics has been a subject of immense interest over the last few decades and has been termed as the thermodynamic formalism of complex systems [8]. As discussed earlier, detailed modelling of complex physical processes often proves to be mathematically challenging and computationally intensive especially in the presence of high dimensional phase space. In Statistical Mechanics, similar issue is tackled by estimating the macroscopic properties (e.g., pressure and temperature) of the entire system from the distribution of the elementary particles in various microstates. In the same fashion, the behavior of a dynamical system can be investigated both from the microscopic and the macroscopic points of view. From the perspective of Statistical Mechanics, the set of time series data can be conceptually visualized to be analogous to a thermodynamic system, where each data point can be treated as a particle in the Statistical Mechanical sense. The global macroscopic behavior of the system can be estimated from the time series data set by describing statistical distributions of these so called data particles in different energy levels which are defined by partitioning the data space (See Section II-A).

The pattern identification of a quasi-stationary process is recognized as a two-time-scale problem. The Fast-time scale refers to the local behavior of the system and is defined as the time scale over which the dynamical behavior of the system is assumed to remain invariant, i.e., the process has stationary dynamics. From the perspective of Statistical Mechanics, the system stays in equilibrium on the same energy hypersurface in its phase space. In other words, even though, 


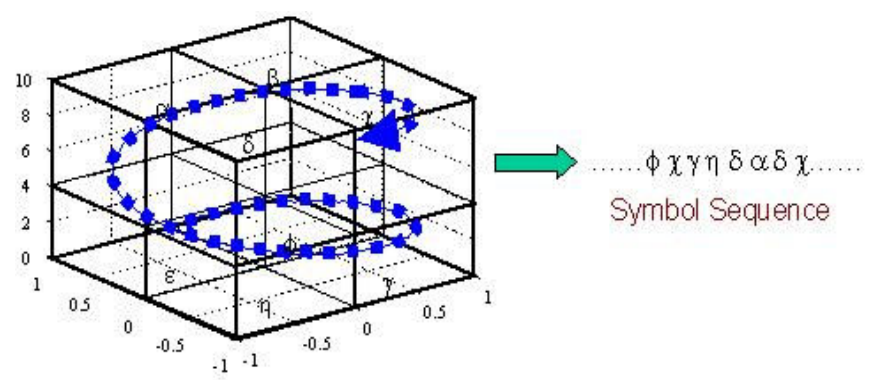

Fig. 1. Concept of phase space partitioning for symbol sequence generation individual particles migrate from one microstate to the other, the probability densities and hence, the macroscopic properties, characteristic of that particular system behavior remain constant over the fast time scale. The Slow-Time Scale, on the other hand, refers to the long-term behavior of the system, where the patterns of the process dynamics might deviate from those under the nominal conditions. It is assumed that any observable non-stationary behavior pattern is associated with changes occurring on the slow time scale. In Statistical Mechanical analogy, the system traverses from one energy hyper-surface to another in between two slow time epochs.

In general, a long time span in the fast time scale is a tiny (i.e., several orders of magnitude smaller) interval in the slow time scale. For example, evolution of fatigue damage in structural materials (causing a detectable change in the dynamics of the system) occurs on the slow time scale (possibly in the order of hours or months); fatigue damage behavior is essentially invariant on the fast time scale (approximately in the order of seconds or minutes). Nevertheless, the notion of fast and slow time scales is dependent on the specific application and operating environment.

\section{A. A Brief Review of Symbolic Dynamic Filtering}

In symbolic dynamic filtering $(S D F)$ procedure [5], a data sequence is converted to a symbol sequence by partitioning a compact region of the phase space of the dynamical system, over which the trajectory evolves, into finitely many discrete blocks (Fig. 1). Each block is labelled as a symbol, where the symbol set $\Sigma$ is called the alphabet that consists of $|\Sigma|$ different symbols. (Note: $|\Sigma| \geq 2$.) As the system evolves in time, it travels through or touches various blocks in its phase space and the corresponding symbol $\sigma \in \Sigma$ is assigned to it, thus converting the data sequence into a symbol sequence.

A crucial step in $S D F$ is partitioning of the phase space for symbol sequence generation. Several partitioning techniques have been reported in literature for symbol generation [9], primarily based on symbolic false neighbors. These techniques may become cumbersome and extremely computation-intensive if the dimension of the phase space is large. Therefore, as an alternative, symbol sequences can be generated from the measured time series data of available sensors [10]. Figure 2 presents an illustrative example of partitioning the time series data, to generate a symbol sequence. The appropriate signal conditioning method using the wavelet transform can be applied to the time series data before partitioning. Since wavelet transform are particularly effective with noisy data from high-dimensional dynamical

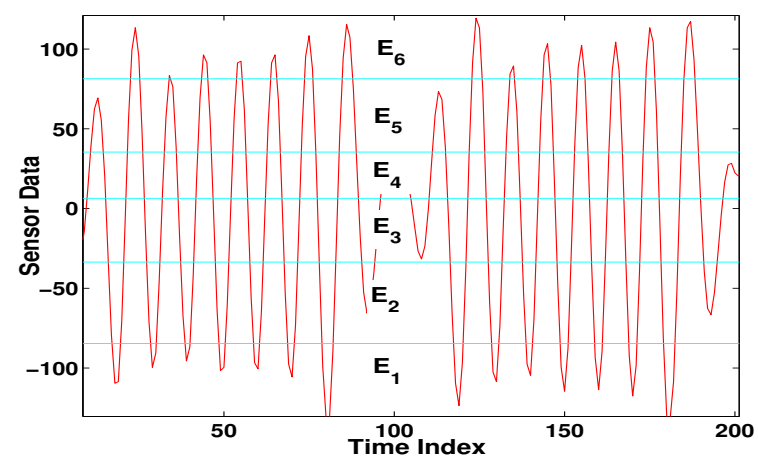

Fig. 2. Illustration of data partitioning for symbol sequence generation

systems, this paper has adopted a wavelet-based partitioning approach [5] [7].

The partitioning is done such that the regions with more information are partitioned finer and those with sparse information are partitioned coarser. This is achieved by maximizing the Shannon entropy, which is defined as $S=$ $-\sum_{i=1}^{|\Sigma|} p_{i} \log \left(p_{i}\right)$ where $p_{i}$ is the probability of the $i^{t h}$ state and summation is taken over all possible states. Each partition region is identified as a state $q_{j} \in Q$. Uniform probability distribution of states is a consequence of maximum entropy that makes the partition coarser in regions of low data density and finer in regions of high data density.

Once the partitioning is done with alphabet size $|\Sigma|$ at the nominal condition (time epoch $t_{0}$ ), it is kept constant for all (slow time) epochs $\left\{t_{1}, t_{2}, \ldots . t_{k} \ldots.\right\}$, i.e. the structure of the partition is fixed at the nominal condition. The probability of transitions from state $q_{j}$ to state $q_{k}$ belonging to the set $Q$ of states under a transition $\delta: Q \times \Sigma \rightarrow Q$ is defined as

$$
\pi_{j k}=P\left(\sigma \in \Sigma \mid \delta\left(q_{j}, \sigma\right) \rightarrow q_{k}\right) ; \sum_{k} \pi_{j k}=1 ;
$$

Thus, the irreducible stochastic matrix $\Pi \equiv\left[\pi_{i j}\right]$ describes all transition probabilities between states. The time series data under the nominal condition is set as the reference point. The state transition matrix $\Pi^{0}$ at $t_{0}$ is generated to obtain the state probability vector $\mathbf{p}^{0}$ whose elements are the stationary probabilities of the state vector, where $\mathbf{p}^{0}$ is the left eigenvector of $\Pi^{0}$ corresponding to the (unique) unity eigenvalue [5]. Subsequently, state probability vectors $\mathbf{p}^{1}, \mathbf{p}^{2}, \ldots \mathbf{p}^{k} \ldots$ are obtained at slow-time epochs $t_{1}, t_{2}, \ldots t_{k} \ldots$ based on the respective time series data.

\section{B. Escort Distributions for Pattern Identification}

In Statistical Mechanics, the equilibrium probability distribution of the energy states, called the Generalized Canonical Distribution, is estimated by maximizing the entropy of the system given a macroscopic parameter, such as the energy $<E>$ [8]. This distribution is obtained using the method of unbiased guess of the probabilities for a given value of the macroscopic parameter. Let $E$ be a random variable which takes a value $E_{j}$ in the energy state $j$ and let $P_{j}$ be the equilibrium probability of that state. The objective is to estimate the equilibrium probability vector $\mathbf{P}$ given a macroscopic 
parameter $\langle E\rangle$ with the following constraints:

$$
\begin{gathered}
<E>=\sum_{j=1}^{|\Sigma|} P_{j} E_{j}, \text { and } \\
\sum_{j=1}^{|\Sigma|} P_{j}=1 .
\end{gathered}
$$

The method of Lagrange multipliers is used [8] for maximization of entropy $S=-\sum P_{j} \ln \left(P_{j}\right)$ while the constraints given by Eqs. (2) and (3) are satisfied. Therefore, the following relations can be derived

$$
\begin{gathered}
\sum_{j=1}^{|\Sigma|} E_{j} \delta P_{j}=0 \\
\sum_{j=1}^{|\Sigma|} \delta P_{j}=0 \text { and } \\
\sum_{j=1}^{|\Sigma|}\left(\ln P_{j}-\Psi+\beta E_{j}\right) \delta P_{j}=0
\end{gathered}
$$

where $\Psi$ and $\beta$ are the Lagrange multipliers. Since $\delta P_{j}$ 's are arbitrary each of the terms in the summation in Eq. (6) must be zero [8]. Therefore, the equilibrium probability distribution, i.e., the Generalized Canonical Distribution [8], is obtained from Eq. (6) as follows:

$$
P_{j}=\exp \left(\Psi-\beta E_{j}\right), j=1, \ldots|\Sigma| .
$$

Using Eq. (3),

$$
P_{j}=\frac{\exp \left(-\beta E_{j}\right)}{\sum_{i=1}^{|\Sigma|} \exp \left(-\beta E_{j}\right)}, j=1, \ldots|\Sigma| .
$$

where the parameter $\beta$ is identified as the inverse of the temperature of the system and the partition function $Z(\beta)=\exp (-\Psi)$ appears as the normalizing factor

$$
Z(\beta)=\sum_{i=1}^{|\Sigma|} \exp \left(-\beta E_{i}\right) .
$$

As described earlier in Section II-A, the time series data is partitioned to generate the symbol sequences and the state probabilities are obtained for each partition state. The objective now is to assign the equilibrium distribution, i.e., the generalized canonical distribution (using Eq. (8)), to the partition states that are analogous to the Statistical Mechanical energy states as shown in Fig. 2. For dynamical systems, as shown in ref [8], the energy states $E_{j}$ 's are related to the state visit probabilities $p_{j}$ 's by:

$$
E_{j}=-\ln p_{j}, j=1, \ldots|\Sigma|,
$$

where $p_{j}$ is observed probability (obtained from partitioning the experimental data using $S D F$ as described in Section IIA) of the $i^{t h}$ energy state. The rational for the above substitution are given below:

- Since $-\ln p_{j} \geq 0$, this implies $0 \leq E_{j} \leq \infty$
- The partition levels that have low frequency of visit (i.e., regions with low information content) have corresponding high energies (less reachable) and the partition levels that have high frequency of visit (i.e., regions with high information content) have corresponding low energies (more reachable) in the Statistical Mechanical sense. For e.g., if state $j$ is never visited, i.e., $p_{j}=0$, then it corresponds to infinite energy state that is never reached by a particle.

Using Eq. (10) in Eq. (8) we get,

$$
P_{j}=\frac{\left(p_{j}\right)^{\beta}}{\sum_{j=1}^{|\Sigma|}\left(p_{i}\right)^{\beta}}, j=1, \ldots|\Sigma| .
$$

which provide the Generalized Canonical distribution $\mathbf{P}$, also called the escort distribution of $\mathbf{p}$ of order $\beta$. The escort distribution $\mathbf{P}$ has the ability to scan the structure of the original probability distribution $\mathbf{p}$. The order $\beta$ that is analogous to the inverse temperature, affects the relative importance of how the microstates $j$ enter into the escort distribution. The escort distribution $\mathbf{P}$ reveals more information of the system than the original probability distribution $\mathbf{p}$.

Since $\beta \sim 1 / T$, where $\mathrm{T}$ is the effective temperature, scanning the probability distribution $\mathbf{p}$ at different values of $\beta$, as shown in Eq. (11), can be interpreted as analogous to a change of temperature in a thermodynamic system. (Note: the analogy presented here is only constructive and not exact). When $\beta \rightarrow 0$, the temperature $\mathrm{T}$ tends to $\infty$ in a thermodynamic sense, which means that the system becomes random and the uncertainty in the system becomes very high such that all the energy states become equally excited. This leads to equal escort probability $\left(P_{j}=1 /|\Sigma|\right.$, $\mathrm{j}=1, \ldots,|\Sigma|)$ of each energy state and due to the maximum uncertainty, the information content obtained from the system is minimized. As the value of $\beta$ is increased, the temperature $\mathrm{T}$ of the system decreases; consequently, the uncertainty is reduced and more information is revealed. Therefore, at lower temperature $\mathrm{T}$ or higher value of $\beta$ many new facets of the system are revealed. As such, the parameter $\beta$ of the escort distribution $\mathbf{P}$ can scan the structure of the state probability vector $\mathbf{p}$ and can reveal information during early stages of fatigue damage evolution.

\section{Damage Evolution and Anomaly Detection}

The anomalies (i.e., deviations of the evolving patterns from the nominal pattern) are characterized by a scalarvalued function, called Anomaly Measure $\psi$ that is quasistatic in the fast time scale and is monotonically nondecreasing in the slow time scale. The escort probability vector at any time epoch corresponds to a singleton point on the unity-radius hypersphere. During fatigue damage evolution, the tip of the escort probability vector moves on the surface of this hypersphere. The initial starting point is the escort probability vector $\mathbf{P}^{0}$ with uniform distribution obtained with maximum entropy partitioning [7]. As the damage progresses, the escort distribution changes; eventually when a very large crack is formed, complete attenuation of the ultrasonic time series data occurs and consequently the tip 
of the escort probability vector reaches a point where all states have zero probabilities of occurrence except one which has a probability one (i.e., a delta-distribution $\mathbf{P}^{f}$ ); this state corresponds to the partition region where all data points are clustered due to complete attenuation of the signal. In the context of an irreversible process such as fatigue damage, the anomaly measure is based on the following assumptions:

- Assumption \#1: The damage evolution is an irreversible process (i.e., with zero probability of self healing) and implies the following conditions.

$$
\psi(t) \geq 0 ; \quad \psi(t+\delta)-\psi(t) \geq 0 \forall t \geq t_{0} \forall \delta>0
$$

- Assumption \#2: The damage accumulation between two time epochs is a path function, i.e., dependent on the path traversed to the target state from the initial state.

At the initial stages of fatigue damage, there can be multiple short cracks oriented in different directions. Therefore, crack length alone does not provide complete information on fatigue damage evolution. Since ultrasonic signals are highly sensitive to small micro-structural changes, signal distortion is a good index of anomaly growth. As such, the following distance function is used between the escort probability vectors at two time epochs:

$$
d\left(\mathbf{P}^{k}, \mathbf{P}^{l}\right) \equiv \sqrt{\left(\mathbf{P}^{k}-\mathbf{P}^{l}\right)^{T}\left(\mathbf{P}^{k}-\mathbf{P}^{l}\right)}
$$

The algorithm for computation of the anomaly measure $\psi$ compensates for spurious measurement and computation noise in terms of the sup norm $\|$ e $\|_{\infty} \equiv$ $\max \left(\left|e_{1}\right|, \cdots,\left|e_{m}\right|\right)$ of the error in the state probability vector (i.e., the maximum error in the elements of the state probability vector). The algorithm is presented below.

i) $\psi^{0}=0 ; \delta \psi^{1}=0 ; \widetilde{\mathbf{P}}=\mathbf{P}^{0} ; \widetilde{\mathbf{p}}=\mathbf{p}^{0} \mathrm{k}=1$;

ii) if $\left\|\mathbf{p}^{k}-\widetilde{\mathbf{p}}\right\|_{\infty}>\epsilon$ then $\delta \psi^{k}=d\left(\mathbf{P}^{k}, \widetilde{\mathbf{P}}\right) ; \widetilde{\mathbf{p}} \leftarrow \mathbf{p}^{k}$ and $\widetilde{\mathbf{P}} \leftarrow \mathbf{P}^{k}$;

iii) $\psi^{k}=\psi^{k-1}+\delta \psi^{k}$;

iv) $k \leftarrow k+1 ; \quad \delta \psi^{k}=0 ; \quad$ go to step (ii).

The real positive parameter $\epsilon$, associated with robustness of the anomaly measure for measurement and computation noise, is identified by performing an experiment with a sample with no notch. Since there is no notch there is practically no stress augmentation and relatively no fatigue damage. As such, the parameter $\epsilon$ is estimated as:

$$
\epsilon \approx \max _{l \in\{1, . . N\}}\left(\left\|\mathbf{p}^{l+1}-\mathbf{p}^{l}\right\|_{\infty}\right)
$$

from $N$ consecutive observations with $N \gg 1$. The algorithm works in the following fashion: the reference point $\widetilde{\mathbf{P}}$ is initialized to the starting point $\mathbf{P}^{0}$ and anomaly measure $\psi^{0}$ is set to 0 . At any slow time epoch $t_{k}$ if the state probability vector moves such that the distance moved in any particular direction (i.e. the sup norm $\|\bullet\|_{\infty}$ ) is greater than $\epsilon$ as specified in step (ii), then the anomaly measure is incremented by $\delta \psi^{k}=d\left(\mathbf{P}^{k}, \widetilde{\mathbf{P}}\right)$ and the reference point is shifted to the current point $\mathbf{P}^{k}$. The procedure is repeated at all slow time epochs.

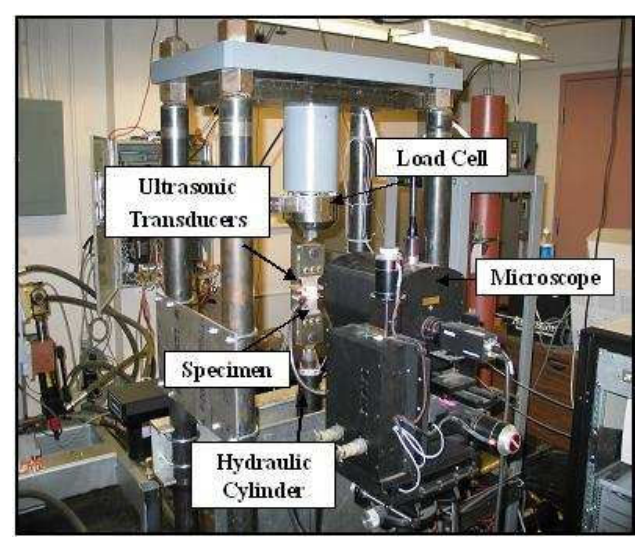

Fig. 3. Special-purpose fatigue test apparatus

\section{Summary of the Steps Followed}

- Time series data acquisition from appropriate sensor(s) at time epoch $t_{0}$, i.e., the nominal condition, when the system is assumed to be in the healthy state (i.e., zero anomaly measure)

- Generation of the maximum entropy partition based on the nominal data set after signal conditioning for noise removal using wavelets [7]. The partitioning is fixed for subsequent time epochs $t_{1}, t_{2}, \ldots t_{k} \ldots$

- Calculation of the state probability vector $\mathbf{p}^{0}$ using $S D F$ (See Section II-A) and the corresponding escort probability vector $\mathbf{P}^{0}$ for different orders $\beta$ (See Section II-B) at time epoch $t_{0} . \mathbf{P}^{0}$ is a uniform distribution because of maximum entropy partitioning [7]

- Collection of time series data at slow time epochs $t_{1}, t_{2}, \ldots t_{k} \ldots$ and calculation of the corresponding escort probability vectors $\mathbf{P}^{1}, \mathbf{P}^{2}, \ldots \mathbf{P}^{k}, \ldots$

- Computation of Anomaly Measures $\psi_{1}, \psi_{2}, \ldots, \psi_{k}, \ldots$, at time epochs $t_{1}, t_{2}, \ldots t_{k} \ldots$ (See Section II-C).

\section{DESCRIPTION OF THE EXPERIMENTAL APPARATUS}

The experimental apparatus, shown in Fig. 3, is a specialpurpose uniaxial fatigue testing machine, which is operated under load control or strain control at speeds up to $12.5 \mathrm{~Hz}$. A typical specimen, made of 7075-T6 aluminum alloy, is shown in Fig. 4. The specimen is $3 \mathrm{~mm}$ thick and $50 \mathrm{~mm}$ wide with a slot on one side of $1.58 \mathrm{~mm}$ diameter and $4.57 \mathrm{~mm}$ length. The notch is made to increase the stress concentration factor in that region and it guarantees crack propagation at notch end. The sensors for damage detection are:

1) Travelling Optical Microscope: The travelling optical microscope (Fig. 3) provides direct measurements of the surface crack. The resolution of the microscope is about 2 microns at a working distance of 10 to $35 \mathrm{~cm}$.

2) Ultrasonic Flaw Detector: The ultrasonic flaw detector functions by emitting high frequency $(5 \mathrm{MHz}$ sine wave) ultrasonic pulses that travel through the specimen and return back through the receiver transducers. The signal is sent through the region of crack propagation and received on the other side. Since material characteristics (e.g., voids, dislocations and short cracks) influence the ultrasonic impedance, a 


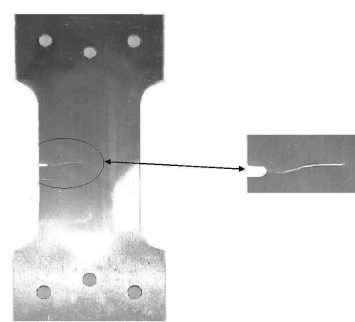

Fig. 4. Cracked specimen with a side notch

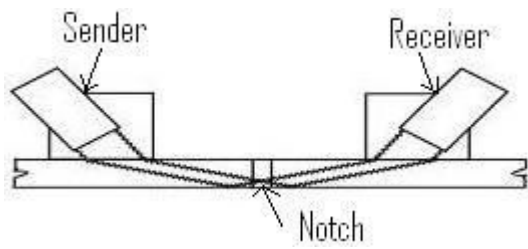

Fig. 5. Ultrasonic flaw detection scheme

small fault in the specimen is likely to change the signature of the signal at the receiver end.

The fatigue tests were conducted at a constant amplitude sinusoidal load for low-cycle fatigue, where the maximum and minimum loads were kept constant at $87 \mathrm{MPa}$ and $4.85 \mathrm{MPa}$, respectively. A significant amount of internal damage caused by multiple small cracks, dislocations and microstructural defects alters the ultrasonic impedance, which results in signal distortion and attenuation at the receiver end. The crack propagation stage starts when this internal damage eventually develops into a single large crack. The slow time epochs for data analysis were chosen to be 1000 load cycles (i.e., $\sim 80 \mathrm{sec}$ ) apart. At the onset of each slow time epoch, the ultrasonic data points were collected on the fast time scale of 50 cycles (i.e., $\sim 4 \mathrm{sec}$ at $12.5 \mathrm{~Hz}$ frequency), which produced a string of 30,000 data points. It is assumed that during this period, the system remained in a stationary condition and no major changes occurred in the fatigue crack behavior. The sets of time series data collected in this manner at different slow-time epochs were analyzed to calculate the anomaly measures at those slow time epochs.

\section{RESUlTS AND DISCUSSION}

The six plots in Fig. 6 show two-dimensional images of the specimen surface and the corresponding escort probability distributions of energy states $(|\Sigma|=6)$, at six different slow time epochs, approximately 1, 10, 18, 23, 32 and 45 kilocycles, exhibiting gradual evolution of fatigue damage. For each time epoch, i.e., each plot in Fig. 6, the escort distribution is shown for different values of $\beta=0,0.5,1,2,5$ and 10 and the top figure exhibits the image of the specimen surface as seen by the microscope. The plot (a) of Fig. 6 shows the image at the nominal condition ( $\sim 1$ kilocycles). The maximum entropy principle used for partitioning has lead to a uniform probability distribution in the energy states as seen in the histograms for all values of order $\beta$. The anomaly measure at this point is taken to be zero. Plots (b) and (c) at $\sim 10$ and $\sim 18$ kilocycles respectively, do not yet have any indication of surface crack but the escort distribution histograms of different orders $\beta$ exhibit deviations from the uniform probability distribution. This is an evidence that the analytical measurements, based on ultrasonic sensor data, produce damage information during the crack initiation stage. The image in plot (d) of Fig. 6 at $\sim 23$ kilocycles exhibits the first noticeable appearance of a small surface crack, this may be considered as the boundary of the crack initiation and the propagation phases. The corresponding histograms in (d) show further deviation from the uniform distribution of plot (a). The image in plate (e) at $\sim 32$ kilocycles exhibits a fully developed crack in its propagation phase. The corresponding histograms show significant variation from those in earlier stages, from (a) to (d). The image in plate (f) at $\sim 45$ kilocycles exhibits a large crack or a broken specimen. The corresponding histogram resembles a delta distribution indicating complete attenuation of the ultrasonic data.

In all the histogram plots in Fig. 6 at different time epochs, the escort probabilities of all energy states are equal for $\beta=$ 0 , irrespective of the system health. This indicates that for $\beta=0$, i.e., $T \rightarrow \infty$, the system attains a maximum entropy state resulting in no information retrieval. In all plots when $\beta$ is increased from $\beta=0$, more information is revealed which is of significant importance especially during the crack initiation. Therefore, higher order escort distribution yield a better picture of the system with distinct probability distributions. For higher order $\beta$, the higher probability states become more prominent and lower probability states become less significant resulting in more sensitivity to small change detection. In all plots of Fig. 6 , as $\beta$ is increased from 0 to 10 , the difference in escort probabilities of different states become more noticeable.

Figure 7 shows the profile of Anomaly Measure representing fatigue damage evolution at different slow time epochs. The anomaly measure profiles are plotted for different values of $\beta=0,0.5,1,5,10$ and 20 . In the region around $\sim 20-23$ kilocycles, a rapid change in the slope of anomaly measure profiles is observed for all $\beta$ that indicates the onset of crack propagation phase (as shown by the vertical line in Fig. 7), after which, the growth of anomaly measure is very fast till complete breakage of the specimen. At $\beta=0$, the profile is zero yielding no information. For $\beta=0.5$ and 1 , the anomaly measure profiles capture the crack propagation phase when the slope of anomaly measure profile is very high; however, the crack initiation phase is not detected significantly, i.e., the anomaly measure profile is incapable of issuing early warning. As $\beta$ is increased, the anomaly measure curve shows a more sensitive response to crack growth, specially in the initiation phase in terms of both the amplitude and the slope of the anomaly measure. For $\beta>1$ the anomaly measure profiles show significant deviations from the nominal condition even during early stages of crack initiation. In the crack propagation region, the profiles of all $\beta$ (except $\beta=0$ ) are sensitive in predicting damage; however, in the crack initiation stage the anomaly measure profiles derived from higher order escort distributions play a significant role in detecting early damage. Increasing $\beta$ at very high values at $\beta>10$ would be advantageous in the initial stages for early detection; however it would make 


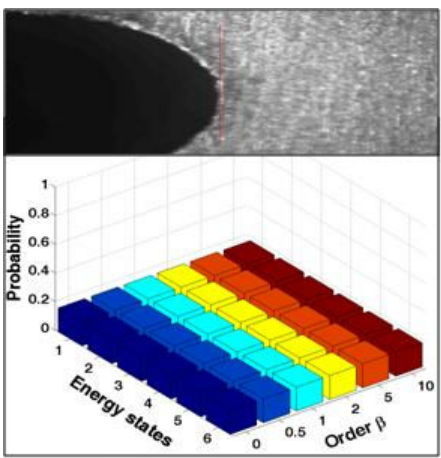

Nominal Condition 1 Kilocycles
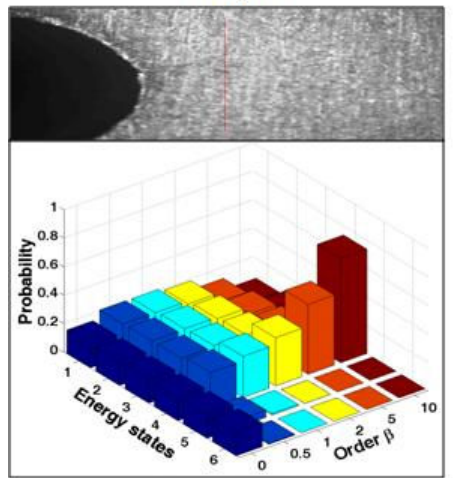

Surface Crack Appearance 23 Kilocycles

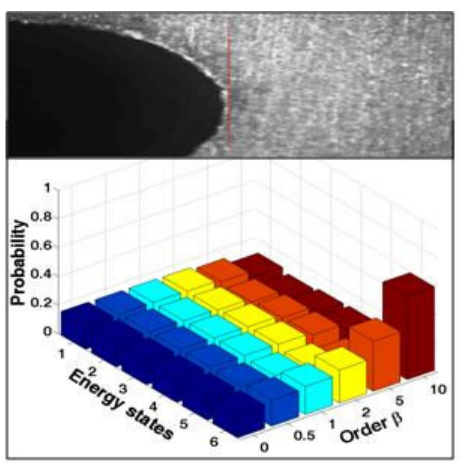

Internally Damaged $\sim 10$ Kilocycles

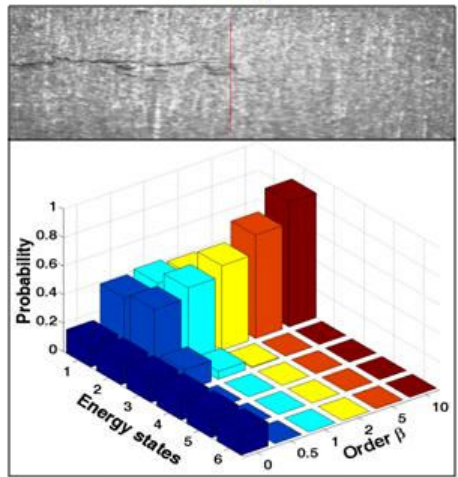

Fully Developed Crack 32 Kilocycles

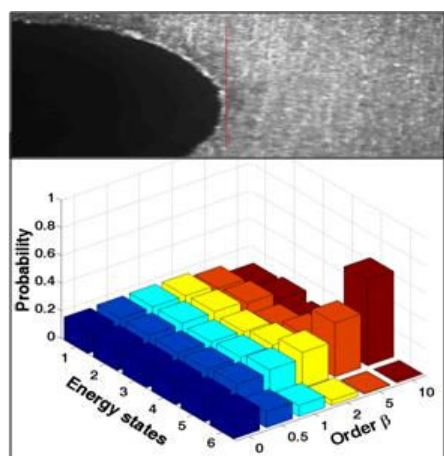

Internally Damaged $\sim 18$ Kilocycles

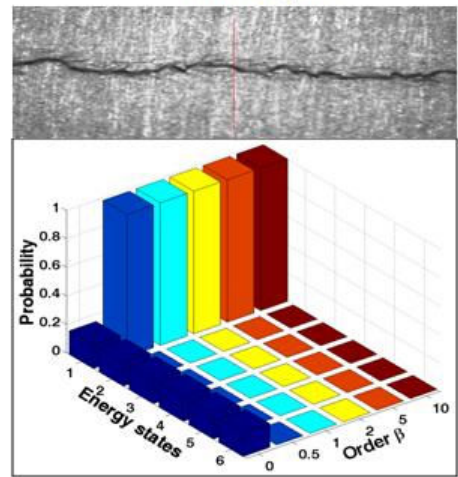

Broken Specimen $\sim 45$ Kilocycles

Fig. 6. Fatigue damage evolution and corresponding information from escort distributions of different order $\beta=0,0.5,1,2,5$ and 10; a) nominal condition at $\sim 1$ kilocycles, b) microstructural damaged condition at $\sim 10$ kilocycles, c) microstructural damaged condition at $\sim 18$ kilocycles, d) appearance of a surface crack at $\sim 23$ kilocycles, e) crack propagation condition with fully developed crack at $\sim 32$ kilocycles, and f) broken condition at $\sim 45$ kilocycles

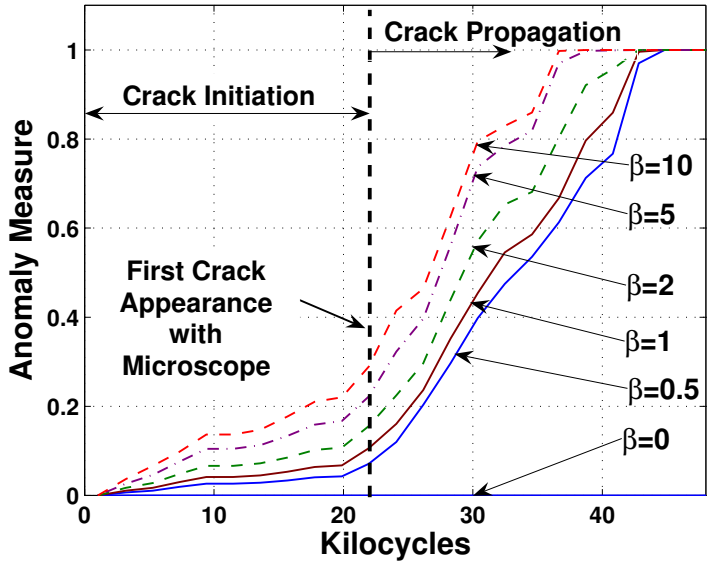

Fig. 7. Anomaly measure profiles for fatigue damage evolution derived from escort distributions of order $\beta=0,0.5,1,2,5$ and 10. the system very sensitive resulting in a sharp jump in the anomaly measure profile for small changes which is not required from the perspective of continuous monitoring of fatigue damage evolution.

\section{CONCLUSIONS}

This paper has presented fatigue damage detection using the ultrasonic data before the onset of widespread damage caused by rapid crack propagation. The real-time data analysis method is based on the concepts of symbolic dynamic filtering and escort distributions derived from Statistical Mechanics. Statistical pattern changes in escort distributions of the observed time series data sequences at different slow time epochs capture the gradual evolution of fatigue damage in poly-crystalline alloys. The concepts have been experimentally validated on a fatigue damage test apparatus.

\section{REFERENCES}

[1] S. Grondel, C. Delebarre, J. Assaad, J. Dupuis, and L. Reithler, "Fatigue crack monitoring of riveted aluminium strap joints by lamb wave analysis and acoustic emission measurement techniques," NDT\&E international, vol. 35, pp. 137-146, 2002.

[2] V. Zilberstein, K. Walrath, D. Grundy, D. Schlicker, N. Goldfine, E. Abramovici, and T. Yentzer, "Mwm eddy-current arrays for crack initiation and growth monitoring," International Journal of Fatigue, vol. 25 , pp. 1147-1155, 2003.

[3] S. Rokhlin and J.-Y. Kim, "In situ ultrasonic monitoring of surface fatigue crack initiation and growth from surface cavity," International journal of fatigue, vol. 25, pp. 41-49, 2003.

[4] R. Duda, P. Hart and D. Stork, Pattern Classification, John Wiley \& Sons Inc., 2001.

[5] A. Ray, Symbolic Dynamic Analysis of Complex Systems for Anomaly Detection, Signal Processing, 84, (7), 2004, pp 1115-1130.

[6] S. Chin, A. Ray, and V. Rajagopalan, "Symbolic time series analysis for anomaly detection: A comparative evaluation," Signal Processing, vol. 85, no. 9, pp. 1859-1868, September 2005.

[7] V. Rajagopalan and A. Ray, "Symbolic time series analysis via wavelet-based partitioning," Signal Processing, vol. 86, no. 11, pp. 3309-3320, 2006.

[8] C. Beck and F. Schlogl, Thermodynamics of chaotic systems: an introduction, Cambridge University Press, United Kingdom, 1993.

[9] M.B. Kennel and M. Buhl, Estimating Good Discrete Partitions form Observed Data: Symbolic False Nearest Neighbors, Physical Review E, 91, (8), 2003, pp. 084102.

[10] S. Gupta, A. Khatkhate, E. Keller and A. Ray, Identification of Statistical Patterns in Complex Systems via Symbolic Time Series Analysis, ISA Transactions, vol. 45, no. 4, pp. 477-490, 2006. 